\title{
Instantaneous Bethe-Salpeter Look at the Lightest Pseudoscalar Mesons
}

\author{
Wolfgang Lucha* \\ Institute for High Energy Physics, Austrian Academy of Sciences, Nikolsdorfergasse 18, A-1050 \\ Vienna, Austria \\ E-mail: Wolfgang.Lucha@oeaw.ac.at
}

\section{Franz F. Schöberl}

Faculty of Physics, University of Vienna, Boltzmanngasse 5, A-1090 Vienna, Austria

E-mail: franz.schoeberl@univie.ac.at

Within our description of Goldstone-type pseudoscalar mesons as almost massless bound states of quark and antiquark by a three-dimensional bound-state equation of Bethe-Salpeter origin, taking into account the pointwise behaviour of the full light-quark propagators enables to characterize the effective interquark interactions more precisely than earlier studies exploiting just specific aspects.

XVII International Conference on Hadron Spectroscopy and Structure - Hadron2017

25-29 September, 2017

University of Salamanca, Salamanca, Spain

${ }^{*}$ Speaker. 


\section{Inversion Starting Point: an Instantaneous Bethe-Salpeter Bound-State Equation}

In the spectrum of hadron states, the lightest pseudoscalar mesons, the pions and kaons, occupy a very isolated position: On the one hand, they must be viewed as bound states of a quark-antiquark pair. On the other hand, they represent (nearly massless) Goldstone bosons related to the dynamical chiral symmetry breakdown of quantum chromodynamics (QCD), owing their nonzero masses only to some additional, explicit breaking of the chiral symmetries. Needless to say, both of these aspects should be incorporated in any reasonable description of such mesons within the framework of QCD.

The homogeneous Bethe-Salpeter equation constitutes a Poincaré-covariant framework for the description of bound states within quantum field theory. Problems due to the occurrence of timelike excitations, inherent to fully relativistic formalisms, may be evaded by relying on three-dimensional reductions accomplished, for instance, by assuming all the effective interactions experienced by the bound-state constituents to be, in the center-of-momentum frame of the bound states, instantaneous. Information on these effective interactions, extracted in form of central potentials $V(r), r \equiv|\mathbf{x}|$, may be deduced by inversion of one's bound-state equation [1]. Recently, we did this for the presumably simplest reduction of this kind [2], the Salpeter equation [3], and a straightforward generalization of the latter [4], designed to incorporate more of the Bethe-Salpeter formalism's relativistic nature [5].

In the present context, inversion simply means the reconstruction of the effective interactions in one's bound-state equation from available knowledge about solutions to this very equation. For ease of presentation, let us impose flavour symmetry, by assuming our two bound-state constituents to be some quark and the corresponding antiquark. Information about this required input to the envisaged inversion process may be harvested by exploiting the fact that the gauge invariance of QCD implies, in the chiral limit, an identity [6] relating the quark propagator and the Bethe-Salpeter solution for a massless pseudoscalar meson in its center-of-momentum frame. If one's formalism strictly respects Poincaré covariance, the full propagator $S(p)$ of a fermion of four-momentum $p$ is totally defined by two Lorentz-scalar functions, this fermion's mass $M\left(p^{2}\right)$ and wave-function renormalization $Z\left(p^{2}\right)$ :

$$
S(p)=\frac{\mathrm{i} Z\left(p^{2}\right)}{\not p-M\left(p^{2}\right)+\mathrm{i} \varepsilon}, \quad \not p \equiv p^{\mu} \gamma_{\mu}, \quad \varepsilon \downarrow 0 .
$$

The semirelativistic bound-state equation constructed in Ref. [5] as an instantaneous approximation to the Bethe-Salpeter formalism poses an implicit eigenvalue problem, with the bound-state masses $\widehat{M}$ as eigenvalues. Its solutions, the Salpeter amplitudes $\phi(\mathbf{p})$, encode the distribution of the relative three-momenta $\mathbf{p}$ of the involved bound-state constituents. For pseudoscalar bound states of spin- $\frac{1}{2}$ fermions, the Salpeter amplitude $\phi(\mathbf{p})$ involves only two independent component functions $\varphi_{1,2}(\mathbf{p})$ :

$$
\phi(\mathbf{p})=\left[\varphi_{1}(\mathbf{p}) \frac{\gamma_{0}\left[\gamma \cdot \mathbf{p}+M\left(p^{2}\right)\right]}{\sqrt{\mathbf{p}^{2}+M^{2}\left(\mathbf{p}^{2}\right)}}+\varphi_{2}(\mathbf{p})\right] \gamma_{5} .
$$

Assuming for the effective interactions Fierz and spherical symmetry, our bound-state equation gets recast to a coupled set of one integral and one algebraic relation [7] for the radial functions $\varphi_{1,2}(|\mathbf{p}|)$ :

$$
\begin{aligned}
& 2 \sqrt{\mathbf{p}^{2}+M^{2}\left(\mathbf{p}^{2}\right)} \varphi_{2}(|\mathbf{p}|)+\frac{4}{\pi} \frac{Z^{2}\left(\mathbf{p}^{2}\right)}{|\mathbf{p}|} \int_{0}^{\infty} \mathrm{d}|\mathbf{q}||\mathbf{q}| \int_{0}^{\infty} \mathrm{d} r \sin (|\mathbf{p}| r) \sin (|\mathbf{q}| r) V(r) \varphi_{2}(|\mathbf{q}|)=\widehat{M} \varphi_{1}(|\mathbf{p}|), \\
& 2 \sqrt{\mathbf{p}^{2}+M^{2}\left(\mathbf{p}^{2}\right)} \varphi_{1}(|\mathbf{p}|)=\widehat{M} \varphi_{2}(|\mathbf{p}|) .
\end{aligned}
$$




\section{Inversion: Confining Interquark Potential Compatible with Goldstone's Theorem}

In the exact Goldstone limit $\widehat{M}=0$, immediately ensuring $\varphi_{1}(|\mathbf{p}|)=0$, the set (1.1) collapses to a single integral equation for the sole surviving component $\varphi_{2}(|\mathbf{p}|) \not \equiv 0$. From the Fourier transform of that latter relation, the sought configuration-space potential $V(r)$ is easily read off. We extract our inversion input from a popular chiral-limit model solution [8] to the QCD equation of motion for the full quark propagator. Figure 1 illustrates our finding for $\varphi_{2}$ in configuration and momentum space. ${ }^{1}$ Using this in the Fourier transform of Eq. (1.1), the behaviour of $V(r)$ given in Fig. 2 can be derived, regrettably only in numerical form, but, at least within the range of $r$ depicted in Fig. 2, can be easily represented in terms of elementary functions, e.g., with the parameter values collected in Table 1, by

$$
V(r) \approx a_{0}+a_{1} r+a_{2} r^{2}+b \exp (c r) .
$$

Table 1: Numerical values of the parameters in our approximation (2.1) to the potential $V(r)$ shown in Fig. 2.

\begin{tabular}{lccccc}
\hline \hline Parameter & $a_{0}[\mathrm{GeV}]$ & $a_{1}\left[\mathrm{GeV}^{2}\right]$ & $a_{2}\left[\mathrm{GeV}^{3}\right]$ & $b[\mathrm{GeV}]$ & $c[\mathrm{GeV}]$ \\
\hline Value & -3.229 & 1.0095 & -0.083685 & $1.411 \times 10^{-5}$ & 0.8475 \\
\hline \hline
\end{tabular}

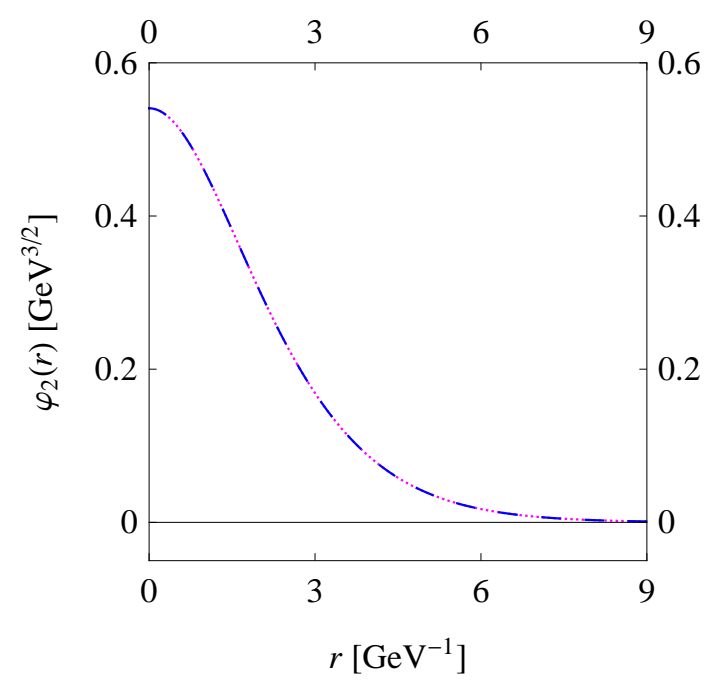

(a)

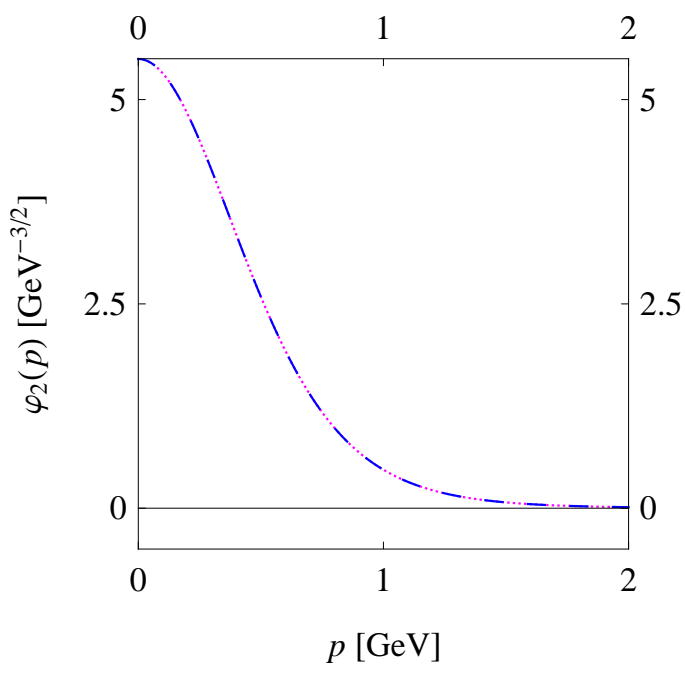

(b)

Figure 1: Nonvanishing radial Salpeter component function (dashed) in configuration (a) and momentum (b) space [4], perfectly matching the $\widehat{M}=0$ solution (dotted) to our three-dimensional bound-state equation (1.1) with the inversion-rooted potential $V(r)$ depicted in Fig. 2 [5], solved by application of variational techniques (a) or conversion to an equivalent matrix eigenvalue problem (b). Here, $p$ indicates the radial variable $p \equiv|\mathbf{p}|$.

\footnotetext{
${ }^{1}$ The predicted meson size makes sense [4]: average interquark distance $\langle r\rangle=0.483 \mathrm{fm}$ and root-mean-square radius $\sqrt{\left\langle r^{2}\right\rangle}=0.535 \mathrm{fm}$ nicely match the measured electromagnetic charge radius $\sqrt{\left\langle r_{\pi}^{2}\right\rangle}=(0.672 \pm 0.008) \mathrm{fm}$ [9] of the pion.
} 


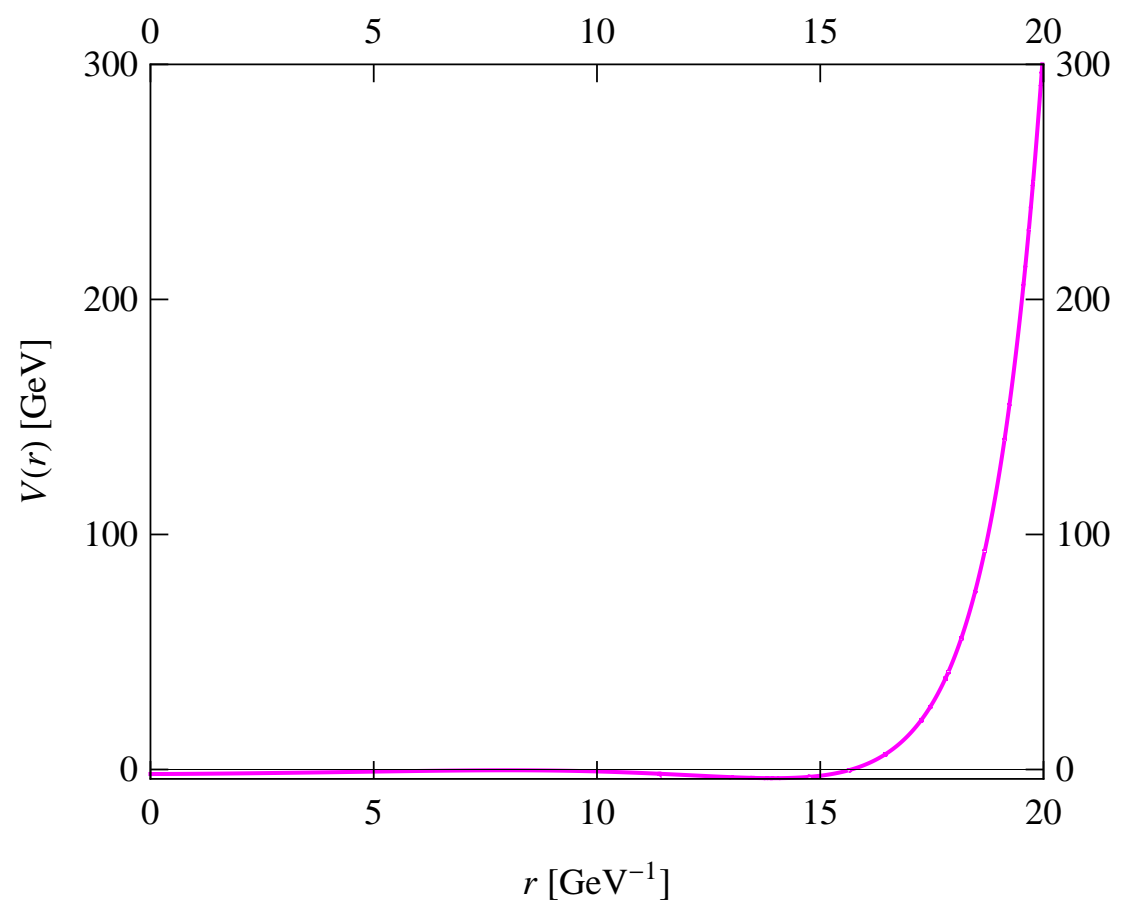

Figure 2: Potential $V(r)$ [4] defining Fierz-symmetric effective interactions providing a proper description of Goldstone-type pseudoscalar mesons by the (three-dimensional) bound-state equation formulated in Ref. [5]: rising in a confining manner from a finite value, $V(0)=-1.92 \mathrm{GeV}$, after passing a zero at $r=15.7 \mathrm{GeV}^{-1}$ to infinity, it resembles, in contrast to earlier findings [2] focusing on particular aspects, a smoothed square well.

\section{References}

[1] W. Lucha and F. F. Schöberl, Phys. Rev. D 87 (2013) 016009, arXiv:1211.4716 [hep-ph]; W. Lucha, Proc. Sci., EPS-HEP 2013 (2013) 007, arXiv:1308.3130 [hep-ph].

[2] W. Lucha and F. F. Schöberl, Phys. Rev. D 92 (2015) 076005, arXiv:1508.02951 [hep-ph]; Phys. Rev. D 93 (2016) 056006, arXiv:1602.02356 [hep-ph]; Phys. Rev. D 93 (2016) 096005, arXiv:1603.08745 [hep-ph]; W. Lucha, EPJ Web Conf. 129 (2016) 00047, arXiv:1607.02426 [hep-ph].

[3] E. E. Salpeter, Phys. Rev. 87 (1952) 328.

[4] W. Lucha and F. F. Schöberl, Int. J. Mod. Phys. A 31 (2016) 1650202, arXiv:1606.04781 [hep-ph]; W. Lucha, EPJ Web Conf. 137 (2017) 13009, arXiv:1609.01474 [hep-ph]; W. Lucha and F. F. Schöberl, Proc. Sci., EPS-HEP2017 (2017) 741, arXiv:1709.02130 [hep-ph].

[5] W. Lucha and F. F. Schöberl, J. Phys. G 31 (2005) 1133, arXiv:hep-th/0507281; Z.-F. Li, W. Lucha, and F. F. Schöberl, Mod. Phys. Lett. A 21 (2006) 1657, arXiv:hep-ph/0510372.

[6] P. Maris, C. D. Roberts, and P. C. Tandy, Phys. Lett. B 420 (1998) 267, arXiv:nucl-th/9707003.

[7] Z.-F. Li, W. Lucha, and F. F. Schöberl, Phys. Rev. D 76 (2007) 125028, arXiv:0707.3202 [hep-ph].

[8] P. Maris and P. C. Tandy, Phys. Rev. C 60 (1999) 055214, arXiv:nucl-th/9905056; P. Maris, in Proc. Int. Conf. on Quark Confinement and the Hadron Spectrum IV, eds. W. Lucha and K. Maung Maung (World Scientific, Singapore, 2002), p. 163, arXiv:nucl-th/0009064.

[9] C. Patrignani et al. (Particle Data Group), Chin. Phys. C 40 (2016) 100001. 